\title{
Metabolic syndrome following liver transplantation in nonalcoholic steatohepatitis
}

\author{
Zachary Spiritos, Manal F. Abdelmalek \\ Division of Gastroenterology and Hepatology, Duke University, Durham, NC, USA \\ Contributions: (I) Conception and design: All authors; (II) Administrative support: MF Abdelmalek; (III) Provision of study materials or patients: \\ None; (IV) Collection and assembly of data: All authors; (V) Data analysis and interpretation: All authors; (VI) Manuscript writing: All authors; (VII) \\ Final approval of manuscript: All authors. \\ Correspondence to: Manal F. Abdelmalek, MD, MPH. Division of Gastroenterology \& Hepatology, 40 Duke Medicine Circle, Basement Level, Orange \\ Zone, Suite 03127, Duke University Medical Center, Durham, NC, USA. Email: manal.abdelmalek@duke.edu.
}

\begin{abstract}
Metabolic syndrome is a major clinical disorder involving metabolic dysregulation characterized clinically with features of central obesity, insulin resistance (IR), type 2 diabetes, hypertension, and dyslipidemia. Metabolic syndrome is strongly associated with the rising prevalence nonalcoholic steatohepatitis, a leading indication for orthotopic liver transplantation in the Western world. The presence or recurrence of metabolic syndrome following liver transplantation can contribute to the development and recurrence of nonalcoholic fatty liver disease (NAFLD) in the liver allograft. In this review, we discuss the endogenous and exogenous drivers of post-transplant metabolic syndrome, role of chronic immunosuppression, and the prevalence and clinical significant of post-transplant metabolic syndrome on nonalcoholic steatohepatitis.
\end{abstract}

Keywords: Cardiovascular disease (CVD); diabetes mellitus; dyslipidemia; hypertension; immunosuppression; obesity; nonalcoholic fatty liver disease (NAFLD); transplant outcomes

Received: 22 August, 2019; Accepted: 16 October, 2019; Published: 05 January 2021.

doi: $10.21037 / \operatorname{tgh} .2020 .02 .07$

View this article at: http://dx.doi.org/10.21037/tgh.2020.02.07

\section{Introduction}

Metabolic syndromes (METs) is a constellation of metabolic derangements that is well known to increase patient risk for nonalcoholic fatty liver disease (NAFLD), nonalcoholic steatohepatitis with progressive hepatic fibrosis and cirrhosis (1). Those patients who developed advance hepatic fibrosis due to NASH are at increased risk for liver-related morbidity and mortality (2). METs is also associated with an increased risk for type 2 diabetes mellitus (T2DM), cardiovascular disease (CVD), hyperuricemia, cholelithiasis, polycystic ovarian syndrome and obstructive sleep apnea (3). The pathophysiology of METs is based on the presence of insulin resistance (IR). As defined by the Adult Treatment Panel III, METs is the presence of 3 or more of the following metabolic derangements: abdominal obesity (waist circumference $>102 \mathrm{~cm}$ in men, $88 \mathrm{~cm}$ in women), hypertriglyceridemia $>150 \mathrm{mg} / \mathrm{dL}, \mathrm{HDL}<40 \mathrm{mg} / \mathrm{dL}$ in men and $<50 \mathrm{mg} / \mathrm{dL}$ in women, hypertension $>130 / 85 \mathrm{mmHg}$ or fasting glucose levels $>110 \mathrm{mmol} / \mathrm{L}(4,5)$.

With improved surgical techniques and optimization of immunosuppression practices, orthotopic liver transplant (OLT) has become a life-saving intervention for patients with complications of end-stage liver disease or acute liver failure. Long-term outcomes of OLT demonstrate impressive results with greater than $90 \%$ survival rate at 1 -year and $50-60 \%$ at 10 years (6). Longer survival postOLT affords the recurrence of pre-transplant METs and its associated complications, including recurrent liver disease due to NAFLD/NASH, and cardiovascular morbidity and mortality comparable to that of the general population. After graft failure and malignancy, CVD is the leading cause of death one year after OLT (7).

With the advent of highly effective and curative direct- 
acting antiviral therapies for hepatitis $\mathrm{C}(\mathrm{HCV}), \mathrm{NASH}-$ related cirrhosis has become the second leading indication for liver transplantation (8). NAFLD, along with its comorbidities, including obesity, T2DM, and the METs, is thus an unmitigated and evolving public health crisis that have only begun to be realized. The percentages of patient on the waitlist or receiving liver transplant for NASH are steadily increasing (9). NASH is projected to be the leading indication for liver transplantation by $2025(9,10)$.

METs disproportionally affect patients after OLT regardless of cirrhosis etiology compared to age-matched population controls (11). The reason for this is multifactorial and is likely due to physiologic changes after OLT and immunosuppressive medications. While the prevalence of METs is estimated to affect $24 \%$ of the adults in the United States (12), approximately $64-71 \%$ of patients with NASH are reported to meet criteria for METs $(13,14)$. Patients transplanted for the indication of NASH cirrhosis are at even higher risk for developing post-transplant METs (PTMETs) given their pre-transplant metabolic co-morbidities (15). Such an at-risk population for CVD requires aggressive pre- and post-transplant screening and management of any metabolic derangements.

METs are common among liver transplant recipients before and after transplantation. The components of METs are often exacerbated in the post-transplant period by transplant specific factors, such as immunosuppression, and are strong predictors of patient morbidity and mortality. Many aspects of the metabolic syndrome are modifiable. Early recognition, prevention and treatment of posttransplant hypertension, obesity, dyslipidemia and diabetes may impact long-term post-transplant survival (16). Here, we review the pathogenesis, epidemiology, risk factors and treatment for PTMETs and its individual components in patients transplanted for complications of NASH cirrhosis.

\section{Endogenous risk factors for PTMETs in NASH}

\section{Post-transplant physiology and homeostasis}

Post-transplant weight gain and obesity are endogenous risk factors for PTMETs and NASH. Cirrhotic patients often develop significant protein-calorie malnutrition due to a chronic hypermetabolic state, fatigue, poor appetite, sarcopenia and ascites accumulation-all of which contribute to impaired functional status $(5,17,18)$. Cirrhotic patients are encouraged to eat a high calorie diet in response to their increased metabolic needs (17). Post-OLT patients not only regain their appetite and recover their nutritional status, but up to $42 \%$ of patients become obese (4).

While increased dietary intake and steroid immunosuppression may contribute to post-transplant weight gain, surgical factors may also play a role in energy homeostasis and increased risk for post-transplant obesity. The liver functions as a metabolic sensor that relays humoral and neuronal signals between the brainstem and the hypothalamus in its regulation of feeding behavior (19). The transection of the native liver's autonomic nerves below the porta hepatitis or at the hepatoduodenal ligament results in the loss of hepatic afferent and efferent neural input which may alter host energy homeostasis and contribute to obesity (6). Studies have shown that liver transplant recipients have higher fat intake, hyperphagia, loss of thermogenesis despite increasing body mass and a decrease in resting energy expenditure (19).

\section{Exogenous risk factors for PTMETs in NASH}

The advent of calcineurin inhibitors (CNIs) such as tacrolimus (TAC) and cyclosporine (CsA) and mammalian target of rapamycin (mTOR) inhibitors have significantly improved allograft success and post-transplant survival (20). Despite the benefits of these medications, they can induce metabolic derangements such as glucose intolerance, dyslipidemia and hypertension. Whether these novel agents affect patient weight remains uncertain.

\section{Immunosuppression therapy}

\section{CNIs}

Immunosuppressive therapy can have deleterious effects on metabolic parameters (Table 1). CsA and TAC are both diabetogenic. CNI's lead to decreased insulin secretion, increased IR and inhibition of steroid metabolism in a dose dependent manner (Figure 1). Decreased insulin secretion may be due to direct B-cell toxicity via vacuolization, degranulation or through inhibition of transcription factors that modulate B-cell growth (20,21). This has been borne out in both animal models and clinical trials. In observational studies TAC appears to be more diabetogenic than CsA (21).

Although both CsA and TAC are prolipidemic, CsA appears to be more deleterious than TAC (22). CsA has been associated with higher levels of triglycerides, total cholesterol, LDL-C and HDL-C compared with TAC in heart transplant recipients (23). The specific dyslipidemic 
Table 1 Deleterious effects of immunosuppression on metabolic parameters

\begin{tabular}{|c|c|c|c|c|}
\hline Metabolic derangement & Corticosteroids* & Tacrolimus $^{1}$ & Cyclosporine $^{1}$ & Rapamune $^{1}$ \\
\hline Obesity & ++ & - & - & - \\
\hline Impaired glucose tolerance & +++ & ++ & + & - \\
\hline Dyslipidemia & ++ & + & ++ & +++ \\
\hline Hypertension & + & + & ++ & - \\
\hline
\end{tabular}

Qualitative depiction of the comparative effects on metabolic parameters by various immunosuppressive agents. *, no comparative data to reference against calcineurin or mTOR inhibitors; ${ }^{1}$, comparative effects as detailed in Reference (21-24). Severity scale: + , mild; ++, moderate; +++, severe. mTOR, mammalian target of rapamycin.

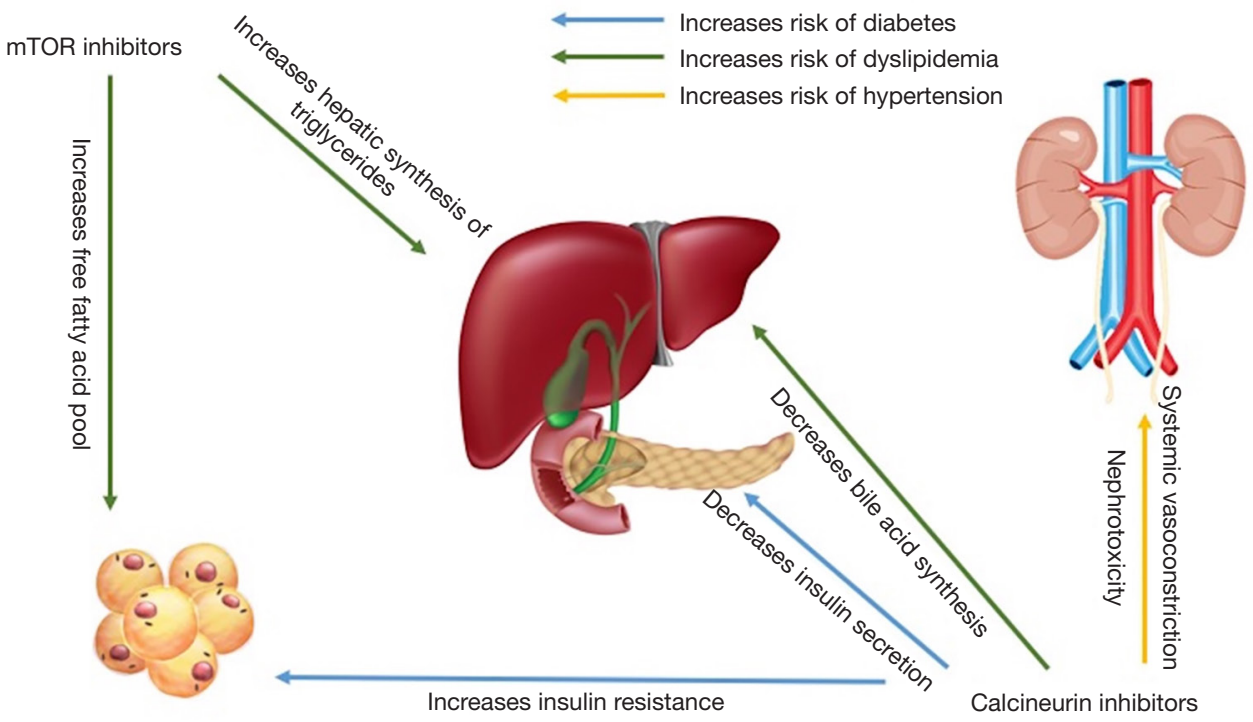

Figure 1 Mechanisms by which calcineurin and mTOR inhibitors negatively affect metabolic parameters. mTOR, mammalian target of rapamycin.

effect of CsA may be due to interference of bile acid synthesis through inhibition of hepatic 26-hydroxylase thereby decreasing cholesterol degredation $(7,22)$.

The effect of CNI's on blood pressure post-OLT is likely multifactorial. Both TAC and CsA cause renal and systemic vasoconstriction which can lead to both nephrotoxicity and elevated blood pressures (4). These agents can increase circulating endothelin-1 (ET-1) a potent systemic vasoconstrictor (24). Studies have documented increased levels of serum and urinary ET-1 up to 2 years after OLT with use of CNI. Interestingly, animal models have shown early success of ET-1 receptor antagonists that could prevent or reverse CNI induced hypertension (24). CsA has been associated with a greater incidence of hypertension compared to TAC. A few reports have suggested an improvement in baseline blood pressure after converting from CsA to TAC (25-27).

\section{mTOR inhibitors}

mTOR inhibitors have more deleterious effects on lipid profiles compared with CNI (23). Specifically, mTOR inhibitor containing immunosuppression regimens lead to significantly elevated triglycerides, LDL-C, total cholesterol and small density-LDL (23). mTOR inhibitors increase free fatty acids which boosts the hepatic synthesis of triglycerides. It is also hypothesized that rapamycin decreases free fatty acid oxidation leading to expansion of the free fatty acid pool (28). Less is known about the diabetogenic, dyslipidemic or hypertensive potential of rapamycin (21). 


\section{Steroids}

Steroids are used in the immediate post-operative setting to prevent allograft rejection. With the advent of alternative steroids-sparing immunosuppression regimens, chronic use of steroids post-OLT is rare. Steroids, either with short- or long-term use, are notorious for causing detrimental effects on metabolism. Steroid use is obesogenic and induces glucose intolerance, hypertension and hyperlipidemia (21). Fortunately, the standard of rapid weaning of steroids in the months following liver transplantation limits these steroidrelated side effects (29).

Acute cellular rejection is the other indication for postoperative steroid use. In modern day practice, the prevalence of acute cellular rejection is only $6 \%$ however these patients require high dose, pulse steroids (30). TAC has been shown to reduce the risk of acute rejection compared to CsA (4). Therefore, more frequent use of steroids with CsA may confound the findings that CsA has proportionally greater effects on dyslipidemia and hypertension. That said, most of these observations have been reproduced even when accounting for concomitant steroid use (31).

\section{Prevalence and patient risk factors for PTMETs in NASH}

Transplant recipients are at increased risk for developing PTMETs. The prevalence of PTMETs amongst liver transplant recipients is estimated to range from $43-59 \%$ overall $(4,15)$. However, among those patients transplanted the indication of NASH-related cirrhosis or cryptogenic cirrhosis, the prevalence of PTMETs is as high as 90\% (30). In comparison, the prevalence of METs amongst ageadjusted general Western patients is 24\% (32). The majority of patients with cryptogenic cirrhosis likely had preexisting $\mathrm{NASH}$ for which the histologic feature of steatohepatitis (hepatitis steatosis with necroinflammation and ballooned hepatocytes) are "burnt-out" leaving only bland features of micronodular cirrhosis (33). Patients with NASH and METs pre-transplant are at increased risk for PTMETs (30).

Older age and NASH cirrhosis independently predict PTMETs for liver transplant recipients (30,32). The former finding has been demonstrated in the non-transplant population where METs increases linearly with age (34). Interestingly, the choice of immunosuppression has reliably been shown not to increase the risk for PTMETs despite their numerous detrimental effects on IR, dyslipidemia and hypertension. Furthermore, higher doses of steroids post-operatively do not seem to increase risk for PTMETs
$(30,32)$. The reason that certain patients develop PTMETs is complex and likely multifactorial; however the lack of a dose-responsive risk of steroids with PTMETs implicates endogenous factors (i.e., genetic factors, pancreatic beta-cell loss, etc.) as the primary reason for this increase risk.

\section{Clinical implication and significance for PTMETs}

\section{Cardiovascular and cerebrovascular disease}

Patients transplanted for NASH, with or without PTMETs, have higher rates of death from CVD compared to patients transplanted for other indications (1) as well as higher risk for cardiovascular and cerebrovascular related morbidity and recurrent NAFLD/NASH (4,5). PTMETs have been shown to exhibit an independent effect on cardiovascular and cerebrovascular morbidity and is a better predictor of these negative clinical outcomes compared to its individual components (35). The prevalence of vascular events such as cerebrovascular accidents, transient ischemia attacks, myocardial infarctions, acute coronary syndrome and sudden cardiac death is higher amongst patients who develop PTMETs. After a follow-up period of 5 years, approximately $30 \%$ of those who developed PTMETs experienced one of the aforementioned vascular events compared to $8 \%$ of patients who did not have PTMETs (5). Despite this risk, patients transplanted for NASH cirrhosis who develop PTMETs have similar survival rates compared to other liver transplant recipients (36).

A recent systematic review found that the 10-year risk of developing a cardiovascular risk after liver transplant for all etiologies was $13.6 \%$. This risk is consistent with a Framingham moderate- to high-risk category (37). Moreover, PTMETs significantly increased that baseline risk compared to transplant recipients without PTMETs $(\mathrm{OR}=4.01)(38)$.

\section{Recurrent NAFLD post-transplant}

Patients who are transplanted for NASH-related cirrhosis almost universally develop post-OLT hepatic steatosis. In one study that compared patients transplanted for NASH and alcoholic cirrhosis, $100 \%$ patients with NASH had evidence of steatosis at 5 years compared to $25 \%$ for the latter cohort (4). Of those patients transplanted for NASH who develop recurrent NAFLD, approximately 9-13\% reportedly progress to NASH (4). Another study showed that $12.5 \%$ of patients transplanted for NASH 
have recurrence that progresses to cirrhosis after a followup period of 28.1 months (39). PTMETs is an independent risk factor for hepatic steatosis (11). Patients with PTMETs develop NAFLD more regularly at 1- and 2-year followup compared to those who did not $(50.0 \%$ vs. $23.0 \%$, and $45.8 \%$ vs. $25.4 \%$ respectively) (11).

\section{Diagnosis and management of PTMETs}

Management of PTMETs should be aimed at prevention and treatment of its individual components. Heightened awareness, screening and patient education are key to identifying those individuals at increased risk for negative clinical outcomes. The approach to management of PTMETs is comparable to management of METs in the non-transplant setting, although health-care providers must take into account relevant and unique aspects of posttransplant physiology (29).

\section{Obesity}

Post-transplant, patients are at increased risk for rapid weight gain. After months of dietary restrictions and a prolonged catabolic condition, cirrhotic patients often become muscle wasted and enter a state of pseudostarvation characterized by sarcopenia, hypoalbuminemia, and protein-calorie malnutrition. OLT reverses the pre-transplant metabolic alterations of catabolism and sarcopenia often leading to significant and rapid weight gain post-transplantation. One study showed that up to $40.7 \%$ of transplant recipients were obese at 1-year, a higher rate of obesity than observed in the general United States adult population (5).

Patient specific risk factors for post-transplant obesity are older age and those who were transplanted for chronic liver disease compared to acute fulminant liver failure. The incidence of obesity is also associated with prior history of excessive weight or higher BMI prior to transplant (5).

Physical exercise to combat obesity is challenging for patients both pre-and post-transplant. Cirrhotic patients are in a chronic hypermetabolic state that leads to fatigue and poor functional status. Post-operatively, 50-75\% of patients remain sedentary (40). Transplant patients are significantly below age-predicted values for exercise capacity and muscle strength as far out as 2.5 years after surgery (40). Even studies that randomized patients to a rigorous exercise regimen showed that these individuals were drastically below sedentary age-predicted exercise capacity (40). Krasnoff et al. hypothesized that cirrhosis in addition to prolonged physical activity prior to transplant causes irreversible muscle dysfunction (40). That said, early exercise and nutritional counseling has led to significant improvement in exercise capacity and therefore lifestyle modification(s) should be implemented early in the transplant process (40).

Experience with pharmacotherapy to assist with weight loss is limited. One prospective trial investigating the use of tetrahydrolipstatin $\left(\right.$ Orlistat $^{\circledR}$ ), a reversible inhibitor of pancreatic lipase, for obese liver transplant patients showed a significant decrease in waist circumference over a 1-year follow-up period without changes in patient weight or BMI with no associated interference with the absorption of TAC (41). No patients in this trial were on CsA or an mTOR inhibitor. Orlistat ${ }^{\circledR}$ appears safe to use in this patient population; however, the efficacy is unclear. Another weight loss agent, sibutramine, a serotonin and noradrenaline reuptake inhibitor, is not recommended for weight loss due to its hepatotoxic potential (41). GLP-1 receptor agonists have been shown to improve glucose control and diabetesrelated comorbidities, including obesity, CVD and chronic kidney disease, in non-transplant patients (42). Although GLP-1 receptor agonists represent a promising therapeutic approach to diabetes after solid-organ transplant, the safety and efficacy of these drugs in transplant recipients have not been adequately studied.

Bariatric surgery has been successfully performed in the transplant setting with good outcomes. The options for bariatric surgery have traditionally been gastric banding, sleeve gastrectomy or Roux-en-Y gastric bypass. Gastric banding is no longer a favored surgical intervention for weight loss due to its relative ineffectiveness (43). As an alternative, sleeve gastrectomy is now typically the first surgical option for a restrictive intervention as it does not interfere with immunosuppression absorption, requires a shorter operative time, leads to durable weight loss and maintains access to the pancreaticobiliary system. Rouxen-Y gastric bypass carries a theoretical risk of drug malabsorption and gastric banding involves placing a foreign prosthesis in an immunosuppressed individual (44).

The timing of bariatric surgery in relation to OLT is also an important consideration. Surgery at the time of OLT limits the need for repeat surgical interventions, particularly once patients are chronically immunosuppressed (44). Such risks include increased risk for infection, postoperative bleeding and/or impaired wound healing. For example, one study demonstrated a $33.3 \%$ complication 
rate after a delayed sleeve gastrectomy including bile leak and dysphagia requiring conversion to a Roux-en- $Y$ gastric bypass (44).

\section{Impaired fasting glucose (IFG)}

IR and impaired glycemic control is common with cirrhosis (45). Potential mechanisms for the increased risk for IR includes reduced glycogen synthesis, decreased glucose oxidation and compromised B-cell function (45). OLT improves insulin sensitivity in only 4-6\% of transplant recipients $(46,47)$. In the majority of patients, post-operative weight gain and the use of diabetogenic immunosuppressive medications exacerbates the risk for developing posttransplant diabetes; therefore, sustained diabetes following OLT is as high as $36.5 \%$ in recipients with a median followup time of 57.2 months (47).

Diabetes following OLT may be due to sustained diabetes or new onset T2DM after transplantation (NODAT). Pre-transplant diabetes often persists after OLT. NODAT typically develops within the first month postOLT (47). Observational reports suggest that NODAT can improve or resolve over time (48). One study showed that approximately $50 \%$ of patients with NODAT improve their glucose tolerance after tapering of immunosuppression (21). Younger age at transplant is predictive of NODAT reversibility (49). Chronic $\mathrm{HCV}$ infection is predictive not only of NODAT but of persistent NODAT (49). Other risk factors for NODAT include TAC, male gender, cumulative dose of steroids and CMV infection (4,21,31,50). Interestingly, patients who receive allografts with the TCFF7L2 polymorphism, a protein that is associated with hepatic glucose production and insulin sensitivity, have a higher susceptibility for developing NODAT (51).

The diagnosis of IFG is made by a fasting glucose of $110-125 \mathrm{mg} / \mathrm{dL}$ or a 2 -hour $75 \mathrm{gm}$ oral glucose tolerance test (OGTT) between 140-199 mg/dL. If either of these tests are positive, they should be repeated to confirm the diagnosis (29). Diabetes is diagnosed if the fasting glucose is greater than $126 \mathrm{mg} / \mathrm{dL}$ or OGTT is greater than $200 \mathrm{mg} / \mathrm{dL}$. Hemoglobin A1C should not be used in the immediate post-operative setting because it is inaccurate until 3 months after OLT due to peri- and post-operative blood transfusions (29). Patients discharged without hyperglycemia should have weekly screening for the first month, then every 3 months and then annually (21).

All patients diagnosed with NODAT should receive adequate patient education about self-management, diet and exercise (21). Although several classes of pharmacotherapy are available for type 2 diabetes, medication-related adverse effects and contraindications such as renal impairment may hamper the ability to achieve optimal glycemic control. Oral agents such as incretins, insulin sensitizers and insulin secretagogues are safe in the post-OLT patient in those recipients with stable cardiac, renal and hepatic function. Post-operative impairments in glomerular filtration rates (GFR) may increase the risk for metformin induced lactic acidosis (4). Glucagon-like peptide-1 (GLP-1) receptor agonists provide a new pharmacotherapeutic option based on the multiple glucose-lowering effects of the human hormone GLP-1 for the treatment of obesity and diabetes (52). Stimulation of pancreatic insulin secretion by GLP-1 receptor agonists is glucose dependent; therefore, GLP-1 receptor agonists confer less risk of hypoglycemia. Primary tolerability issues include transient gastrointestinal symptoms, which can be reduced through dose titration; however, such symptoms could interfere with a patients' ability to take immunosuppression medications (4). Insulin is a safe option as long as patients are educated on its use and can reliably follow-up. This is especially important as immunosuppression is weaned and IR improves (21).

If decreasing the dose of immunosuppression does not help with diabetes management, switching to a different immunosuppressive agent is recommended. For example, patients can be converted from TAC to CsA (21). Converting from TAC to CsA should only be considered if the provider is confident that switching medications will not compromise allograft health. Other options include discontinuing CNI altogether and substituting a mycophenolic acid derivative or an mTOR inhibitor (29).

\section{Dyslipidemia}

Cirrhotic patients rarely suffer from dyslipidemia. Impaired hepatic synthesis of cholesterol and lipid esterification contribute to low cholesterol and triglyceride levels $(3,53)$. However, following transplantation, 66-85\% of liver transplant recipients develop dyslipidemia characterized by elevated cholesterol, triglyceride, sd-LDL and low HDL levels, as early as 1 month post-operatively $(4,5,23)$. mTOR inhibitors tend to cause stronger dyslipidemic effects compared to CNI's. Rapamune reliably worsens hyperlipidemia in a dose-dependent manner (28). Other risk factors for developing post-OLT dyslipidemia include postOLT renal insufficiency (4). Predictors of low HDL levels have not been identified (4), although genetic risk factors 
likely contribute (54).

Dyslipidemia appears to be refractory to dietary changes in the post-OLT population (7). As such, medical practitioners should routinely utilize pharmacotherapy to improve a patient's lipid profile. A large systematic review reported that the 10-year risk of developing a cardiovascular event for post-OLT patients is $13.6 \%$ (38). According to the ACC/AHA practice guidelines, HMG-CoA inhibitors (statins) should be initiated for primary prevention of CVD in those with a predicted 10 -year risk between $5.0 \%$ and $7.5 \%$ (55). Therefore statins should be considered in all post-OLT patients independent of their lipid levels. In fact, several observational studies have demonstrated that statins improve mortality for organ recipients $(29,56,57)$.

When prescribing statin therapy post liver transplant, it is important to consider that CNIs inhibit cytochrome p450 3A4 which metabolizes simvastatin, atorvastatin and lovastatin (21). Therefore, transplant recipients on CNIs taking concomitant statin therapy may be susceptible to potential statin related side effect such as myositis. Pravastatin and fluvastatin are not metabolized by the CYP3A4 isoenzyme and should be prioritized in post-OLT patients $(21,58)$. No data regarding target doses of statins in the post-OLT patients currently exists.

Ezetimibe is another lipid lowering agent that works by inhibiting the enterohepatic circulation of cholesterol. One retrospective analysis showed that ezetimibe monotherapy is effective at lowering LDL levels without adversely affecting allograft function or immunosuppression levels. A theoretical risk of hepatotoxicity when ezetimibe is combined with a statin does exist. Further investigation is required to define the safety of use of ezetimibe in combination with statins, particularly in post-transplant recipients (53).

Fibrates can be considered for those who develop severe hypertriglyceridemia with levels greater than $600 \mathrm{mg} / \mathrm{dL}$ (29). Most fibrates, aside from gemfibrozil, can be nephrotoxic and may be problematic when used with a CNI. Furthermore, fenofibrate has been shown to reduce serum levels of CsA in heart transplant recipients (29). If warranted, fish oil (omega-3) can be safely used as a substitute for fibrate therapy which has few side effects and does not interfere with immunosuppression levels (21).

\section{Hypertension}

Hypertension is the most prevalent post-OLT metabolic disturbance with reported rates of 62-69\% (4) and typically occurs within the first 6 months after transplant. Patients treated with CNI in comparison with mTOR inhibitors tend to develop hypertension more frequently (24). Blood pressure increases can be drastic, with elevations as high as $40-50 \mathrm{mmHg}$ from baseline (25). Patients with a higher MAP prior to transplant are at greater risk for developing post-OLT hypertension, although there is no reported cutoff that confers such increased risk (24).

Calcium channel blockers, such as amlodipine, have been shown to be safe and effective in treating post-OLT hypertension. Caution should be taken with diltiazem, nicardipine and verapamil as these antihypertensive agents can increase CsA levels (59). Although ACE inhibitors are effective in lowering blood pressure, ACE inhibitors can cause hyperkalemia when used concomitantly with CNIs (29). Non-selective beta blockers have been shown to be effective and safe but are not as potent as ACE inhibitors (59).

\section{Features of posttransplant metabolic syndrome (PTMS) as compared to MS}

Whether the natural history and severity of PTMETs differs from METs remains unclear. Existing data suggest that PTMETs may lead to an accelerated atherosclerotic process compared to METs. For example, patients with PTMETs seem to have an unusually early onset of clinically significant atherosclerotic disease. One study reported that patients with PTMETs suffer vascular events such as myocardial infarction, acute coronary syndrome and cerebrovascular accidents at the average age of 57 years old (30). In comparison, the average age of first myocardial infarction for men and women in the general population is 64 and 70 years old respectively (60). Similarly, the reported average age of first stroke is 69 years old (61).

Patients with PTMETs also appear to have a greater frequency of major vascular events compared to those with METs. One study with a 5-year follow-up period reported that $23.2 \%$ of patients with PTMETs had CVD defined as acute coronary syndrome or myocardial infarction and $7.2 \%$ experienced a cerebrovascular event defined as a cerebrovascular attack or transient ischemic attack (30). In a large meta-analysis of the general population, $7.7-17.9 \%$ of patients with METs developed CVD over a follow-up period that ranged from 2.1-8.0 years. The same analysis reported that $2.2-7.4 \%$ of patients with METs developed cerebrovascular disease over a follow-up period that ranged from 2.8-11.0 years (38). Whether the incidences of vascular events are higher in PTMETs patients if they are otherwise 
followed for as long as those with METs remain speculative.

An interesting difference between PTMETs and METs is that the latter has been shown to increase risk for allcause mortality in the general population (RR 1.54, CI: 1.29-1.85) (38). On the other hand, PTMETs has not been shown to affect patient mortality. An important caveat is that published results investigating METs and all-cause mortality followed patients from 1.5-32.7 years. This time frame is substantially larger than the lengthiest longitudinal study analyzing the effect of PTMETs on mortality with a follow-up time of $6.2+/-4.4$ years $(32,38)$. Longer prospective studies are needed to better understand the effect of PTMETs on patient mortality especially as life expectancy improves after transplantation.

\section{Conclusions}

METs are common in patients with NASH and NASHrelated cirrhosis (1). PTMETs in patients with NASHrelated cirrhosis recurs or develops with a reported prevalence as high as $90 \%$ (30). In comparison, the rate of PTMETs in patients transplanted for fulminant liver failure, primary biliary cholangitis, alcoholic cirrhosis is $29 \%, 47 \%$ and $74 \%$ respectively (30).

Post-transplant physiology and immunosuppressive medications contribute, through several complex mechanisms, to the high prevalence of PTMETs. PTMETs has serious clinical implications and significantly increases patient risk for cardiovascular and cerebrovascular disease (38). Medical professionals should therefore aggressively screen transplanted NASH patients for metabolic derangements $(30,32)$ and strive to optimize the metabolic complications (increased weight, glycemic control, dyslipidemia, hypertension etc.) of METs. While there is consensus regarding the need to identify and screen at-risk patients for METs, no practice guidance on the longterm management of patients with PTMETs currently exist. Tailored clinical approaches targeting individual risk factors and/or features in METs in individual patients may lend better improvements in long-term outcomes.

\section{Acknowledgments}

Funding: None.

\section{Footnote}

Provenance and Peer Review: This article was commissioned by the Guest Editors (Sanjaya K Satapathy, David Bernstein, Nitzan Roth) for the series "Liver Transplantation in NASH and ALD" published in Translational Gastroenterology and Hepatology. The article was sent for external peer review organized by the Guest Editors and the editorial office.

Conflicts of Interest: The series "Liver Transplantation in NASH and ALD" was commissioned by the editorial office without any funding or sponsorship. Dr. Abdelmalek receives grant funding paid to her institution for research in NAFLD/NASH from Intercept, Allergan, Genfit, BristolMyers Squibb, NGM Bio, Tiawan J, Madrigal, Gilead, Novo-Nordisk, Poxel, Durect, Inventiva, Viking, Galactin. Dr. Abdelmalek is a scientific advisor for Bristol-Myers Squibb, NGM Bio, Tiawan J, Novo-Nordisk, Inventiva and serves on the speaker's bureau for Alexion and Intercept. Dr. Spiritos has no conflicts of interest to declare.

Ethical Statement: The authors are accountable for all aspects of the work in ensuring that questions related to the accuracy or integrity of any part of the work are appropriately investigated and resolved.

Open Access Statement: This is an Open Access article distributed in accordance with the Creative Commons Attribution-NonCommercial-NoDerivs 4.0 International License (CC BY-NC-ND 4.0), which permits the noncommercial replication and distribution of the article with the strict proviso that no changes or edits are made and the original work is properly cited (including links to both the formal publication through the relevant DOI and the license). See: https://creativecommons.org/licenses/by-nc-nd/4.0/.

\section{References}

1. Vanwagner LB, Bhave M, Te HS, et al. Patients transplanted for nonalcoholic steatohepatitis are at increased risk for postoperative cardiovascular events. Hepatology 2012;56:1741-50.

2. Angulo P, Kleiner DE, Dam-Larsen S, et al. Liver Fibrosis, but No Other Histologic Features, Is Associated With Long-term Outcomes of Patients With Nonalcoholic Fatty Liver Disease. Gastroenterology 2015;149:389-97.e10.

3. Sorice GP, Muscogiuri G, Mezza T, et al. Metabolic syndrome in transplant patients: an academic or a health burden? Transplant Proc 2011;43:313-7.

4. Pagadala M, Dasarathy S, Eghtesad B, et al. Posttransplant 
metabolic syndrome: an epidemic waiting to happen. Liver Transpl 2009;15:1662-70.

5. Anastácio LR, Lima AS, Toulson Davisson Correia MI. Metabolic syndrome and its components after liver transplantation: incidence, prevalence, risk factors, and implications. Clin Nutr 2010;29:175-9.

6. Colle I, Van Vlierberghe H, Troisi R, et al. Transplanted liver: consequences of denervation for liver functions. Anat Rec A Discov Mol Cell Evol Biol 2004;280:924-31.

7. Rossetto A, Bitetto D, Bresadola V, et al. Cardiovascular risk factors and immunosuppressive regimen after liver transplantation. Transplant Proc 2010;42:2576-8.

8. Wong RJ, Aguilar M, Cheung R, et al. Nonalcoholic steatohepatitis is the second leading etiology of liver disease among adults awaiting liver transplantation in the United States. Gastroenterology 2015;148:547-55.

9. Goldberg D, Ditah IC, Saeian K, et al. Changes in the Prevalence of Hepatitis C Virus Infection, Nonalcoholic Steatohepatitis, and Alcoholic Liver Disease Among Patients With Cirrhosis or Liver Failure on the Waitlist for Liver Transplantation. Gastroenterology 2017;152:1090-1099.e1.

10. Charlton MR, Burns JM, Pedersen RA, et al. Frequency and outcomes of liver transplantation for nonalcoholic steatohepatitis in the United States. Gastroenterology 2011;141:1249-53.

11. Sprinzl MF, Weinmann A, Lohse N, et al. Metabolic syndrome and its association with fatty liver disease after orthotopic liver transplantation. Transpl Int 2013;26:67-74.

12. Ford ES, Giles WH, Dietz WH. Prevalence of the metabolic syndrome among US adults: findings from the third National Health and Nutrition Examination Survey. JAMA 2002;287:356-9.

13. Marchesini G, Bugianesi E, Forlani G, et al. Nonalcoholic steatohepatitis in patients cared in metabolic units. Diabetes Res Clin Pract 2004;63:143-51.

14. Kotronen A, Westerbacka J, Bergholm R, et al. Liver fat in the metabolic syndrome. J Clin Endocrinol Metab 2007;92:3490-7.

15. Sookoian S, Pirola CJ. The genetic epidemiology of nonalcoholic fatty liver disease: toward a personalized medicine. Clin Liver Dis 2012;16:467-85.

16. Watt KD, Charlton MR. Metabolic syndrome and liver transplantation: a review and guide to management. J Hepatol 2010;53:199-206.

17. Richards J, Gunson B, Johnson J, et al. Weight gain and obesity after liver transplantation. Transpl Int
2005; 18:461-6.

18. Meeks AC, Madill J. Sarcopenia in liver transplantation: A review. Clin Nutr ESPEN 2017;22:76-80.

19. Richardson RA, Garden OJ, Davidson HI. Reduction in energy expenditure after liver transplantation. Nutrition 2001;17:585-9.

20. Heisel O, Heisel R, Balshaw R, et al. New onset diabetes mellitus in patients receiving calcineurin inhibitors: $\mathrm{a}$ systematic review and meta-analysis. Am J Transplant 2004;4:583-95.

21. Lane JT, Dagogo-Jack S. Approach to the patient with new-onset diabetes after transplant (NODAT). J Clin Endocrinol Metab 2011;96:3289-97.

22. Charco R, Cantarell C, Vargas V, et al. Serum cholesterol changes in long-term survivors of liver transplantation: a comparison between cyclosporine and tacrolimus therapy. Liver Transpl Surg 1999;5:204-8.

23. Zimmermann A, Zobeley C, Weber MM, et al. Changes in lipid and carbohydrate metabolism under mTOR- and calcineurin-based immunosuppressive regimen in adult patients after liver transplantation. Eur J Intern Med 2016;29:104-9.

24. Neal DA, Brown MJ, Wilkinson IB, et al. Mechanisms of hypertension after liver transplantation. Transplantation 2005;79:935-40.

25. Hryniewiecka E, Zegarska J, Paczek L. Arterial hypertension in liver transplant recipients. Transplant Proc 2011;43:3029-34.

26. Canzanello VJ, Schwartz L, Taler SJ, et al. Evolution of cardiovascular risk after liver transplantation: a comparison of cyclosporine A and tacrolimus (FK506). Liver Transpl Surg 1997;3:1-9.

27. Lucey MR, Abdelmalek MF, Gagliardi R, et al. A comparison of tacrolimus and cyclosporine in liver transplantation: effects on renal function and cardiovascular risk status. Am J Transplant 2005;5:1111-9.

28. Morrisett JD, Abdel-Fattah G, Hoogeveen R, et al. Effects of sirolimus on plasma lipids, lipoprotein levels, and fatty acid metabolism in renal transplant patients. J Lipid Res 2002;43:1170-80.

29. Wilkinson A, Davidson J, Dotta F, et al. Guidelines for the treatment and management of new-onset diabetes after transplantation. Clin Transplant 2005;19:291-8.

30. Laryea M, Watt KD, Molinari M, et al. Metabolic syndrome in liver transplant recipients: prevalence and association with major vascular events. Liver Transpl 2007;13:1109-14.

31. Li DW, Lu TF, Hua XW, et al. Risk factors for new onset 
diabetes mellitus after liver transplantation: A metaanalysis. World J Gastroenterol 2015;21:6329-40.

32. Laish I, Braun M, Mor E, et al. Metabolic syndrome in liver transplant recipients: prevalence, risk factors, and association with cardiovascular events. Liver Transpl 2011;17:15-22.

33. Contos MJ, Cales W, Sterling RK, et al. Development of nonalcoholic fatty liver disease after orthotopic liver transplantation for cryptogenic cirrhosis. Liver Transpl 2001;7:363-73.

34. Hildrum B, Mykletun A, Hole T, et al. Age-specific prevalence of the metabolic syndrome defined by the International Diabetes Federation and the National Cholesterol Education Program: the Norwegian HUNT 2 study. BMC Public Health 2007;7:220.

35. Hanouneh IA, Feldstein AE, McCullough AJ, et al. The significance of metabolic syndrome in the setting of recurrent hepatitis $\mathrm{C}$ after liver transplantation. Liver Transpl 2008;14:1287-93.

36. Malik SM, Ahmad J. Outcomes of liver transplantation in patients with cirrhosis due to nonalcoholic steatohepatitis versus patients with cirrhosis due to alcoholic liver disease. Liver Transpl 2010;16:533.

37. Lloyd-Jones DM, Wilson PW, Larson MG, et al. Framingham risk score and prediction of lifetime risk for coronary heart disease. Am J Cardiol 2004;94:20-4.

38. Madhwal S, Atreja A, Albeldawi M, et al. Is liver transplantation a risk factor for cardiovascular disease? A meta-analysis of observational studies. Liver Transpl 2012;18:1140-6.

39. Charlton M, Kasparova P, Weston S, et al. Frequency of nonalcoholic steatohepatitis as a cause of advanced liver disease. Liver Transpl 2001;7:608-14.

40. Krasnoff JB, Vintro AQ, Ascher NL, et al. A randomized trial of exercise and dietary counseling after liver transplantation. Am J Transplant 2006;6:1896-1905.

41. Cassiman D, Roelants M, Vandenplas G, et al. Orlistat treatment is safe in overweight and obese liver transplant recipients: a prospective, open label trial. Transpl Int 2006;19:1000-5.

42. Lee YH, Lee HW, Choi HJ. GLP-1 Based Combination Therapy for Obesity and Diabetes. J Obes Metab Syndr 2017;26:155-60.

43. Kowalewski PK, Olszewski R, Kwiatkowski A, et al. Life with a Gastric Band. Long-Term Outcomes of Laparoscopic Adjustable Gastric Banding-a Retrospective Study. Obes Surg 2017;27:1250-3.

44. Diwan TS, Rice TC, Heimbach JK, et al. Liver
Transplantation and Bariatric Surgery: Timing and Outcomes. Liver Transpl 2018;24:1280-7.

45. Perseghin G, Mazzaferro V, Sereni LP, et al. Contribution of reduced insulin sensitivity and secretion to the pathogenesis of hepatogenous diabetes: effect of liver transplantation. Hepatology 2000;31:694-703.

46. Reuben A. Long-term management of the liver transplant patient: diabetes, hyperlipidemia, and obesity. Liver Transpl 2001;7:S13-21.

47. Moon JI, Barbeito R, Faradji RN, et al. Negative impact of new-onset diabetes mellitus on patient and graft survival after liver transplantation: Long-term follow up. Transplantation 2006;82:1625-8.

48. Navasa M, Bustamante J, Marroni C, et al. Diabetes mellitus after liver transplantation: prevalence and predictive factors. J Hepatol 1996;25:64-71.

49. Ahn HY, Cho YM, Yi NJ, et al. Predictive factors associated with the reversibility of post-transplantation diabetes mellitus following liver transplantation. J Korean Med Sci 2009;24:567-70.

50. Delgado-Borrego A, Liu YS, Jordan SH, et al. Prospective study of liver transplant recipients with $\mathrm{HCV}$ infection: evidence for a causal relationship between $\mathrm{HCV}$ and insulin resistance. Liver Transpl 2008;14:193-201.

51. Ling Q, Xie H, Lu D, et al. Association between donor and recipient TCF7L2 gene polymorphisms and the risk of new-onset diabetes mellitus after liver transplantation in a Han Chinese population. J Hepatol 2013;58:271-7.

52. Aroda VR, Ratner R. The safety and tolerability of GLP1 receptor agonists in the treatment of type 2 diabetes: a review. Diabetes Metab Res Rev 2011;27:528-42.

53. Almutairi F, Peterson TC, Molinari M, et al. Safety and effectiveness of ezetimibe in liver transplant recipients with hypercholesterolemia. Liver Transpl 2009;15:504-8.

54. Weissglas-Volkov D, Pajukanta P. Genetic causes of high and low serum HDL-cholesterol. J Lipid Res 2010;51:2032-57.

55. Goff DC Jr, Lloyd-Jones DM, Bennett G, et al. 2013 ACC/AHA guideline on the assessment of cardiovascular risk: a report of the American College of Cardiology/ American Heart Association Task Force on Practice Guidelines. Circulation 2014;129:S49-73.

56. Wenke K, Meiser B, Thiery J, et al. Simvastatin initiated early after heart transplantation: 8-year prospective experience. Circulation 2003;107:93-7.

57. Cosio FG, Pesavento TE, Pelletier RP, et al. Patient survival after renal transplantation III: the effects of statins. Am J Kidney Dis 2002;40:638-43. 
58. Rowan CG, Brunelli SM, Munson J, et al. Clinical importance of the drug interaction between statins and CYP3A4 inhibitors: a retrospective cohort study in The Health Improvement Network. Pharmacoepidemiol Drug Saf 2012;21:494-506.

59. Neal DA, Brown MJ, Wilkinson IB, et al. Hemodynamic effects of amlodipine, bisoprolol, and lisinopril in hypertensive patients after liver transplantation.

doi: $10.21037 / \operatorname{tgh} .2020 .02 .07$

Cite this article as: Spiritos Z, Abdelmalek MF. Metabolic syndrome following liver transplantation in nonalcoholic steatohepatitis. Transl Gastroenterol Hepatol 2021;6:13.
Transplantation 2004;77:748-50.

60. Roger VL, Go AS, Lloyd-Jones DM, et al. Executive summary: heart disease and stroke statistics--2012 update: a report from the American Heart Association. Circulation 2012;125:188-97.

61. Kissela BM, Khoury JC, Alwell K, et al. Age at stroke: temporal trends in stroke incidence in a large, biracial population. Neurology 2012;79:1781-7. 